\title{
Éditorial collaboration spéciale : Introduction au numéro spécial commémorant le 25 ième anniversaire de la création en 1986 de la Série de Butterworth sur le vieillissement individuel et de la population et le 30 ième anniversaire de la fondation de La Revue canadienne du vieillissement
}

Dans le milieu des années 1980, Barry D. McPherson a conçu l'idée d'une série sur le vieillissement individuel et de la population et, après de nombreuses discussions et notes, a convaincu le personnel de Butterworths Publishers que le projet avait du mérite. En conséquence, le Dr McPherson a commencé à modifier un certain nombre de monographies connues collectivement sous le nom Perspectives de Butterworths sur le vieillissement individuel et le viellissement de la population. La première monographie de cette série a été publiée en 1986.

Au moment où cette série a connu une fin prématurée en 1992, un total de 15 monographies ont été publiées. L'année 2011 marque le 25 ième anniversaire du premier volume publié dans la série de Butterworths.

En outre, 2011 marque également le 30 ième anniversaire de la fondation de La Revue canadienne du viellissement. De plus, l'année 2011 est importante pour une autre raison-c'est l'année que la cohorte du baby-boom gagne 65 ans. Par conséquent, ce numéro spécial présente un examen en temps opportun de divers sujets en gérontologie sociale.

La gérontologie sociale était un domaine d'étude qui était relativement nouveau au milieu des années 1980 au Canada et la recherche canadienne était en retard comparée avec celle des États-Unis et du Royaume-Uni. La série de Butterworths a joué un rôle important dans le développement de la gérontologie au Canada.

Chaque volume de la série originale comprenait une revue approfondie des connaissances sur un thème choisi et identifiait des questions sans réponses de politique et de la recherche afin de stimuler les travaux dans le domaine. De même, chaque article dans ce numéro spécial de La Revue canadienne du vieillissement fait la critique du travail accompli sur un sujet donné, depuis la publication de la monographie originale et identifie les politiques et les questions de recherche clés à traiter dans la deuxieme décennie du XXI ième siècle.

Ce numéro spécial marque le début $\mathrm{du}$ " boom des aînés ", ou la vague des aînés, ou avec l'hyperbole apocalyptique injustifiée, le tsunami des personnes âgées. Le vieillissement de la population en général, et le vieillissement de la cohorte du baby-boom (nés entre 1946 et 1966) en particulier, ont reçu beaucoup d'attention au cours des dernières décennies. Ce numéro spécial de La Revue canadienne sur le vieillissement examine la recherche effectuée sur des thèmes choisis relatifs au vieillissement de la population au cours des dernières décennies et identifie les questions qui doivent être abordées dans la recherche future.

Les auteurs des monographies originelles ont été invités à soumettre des articles à ce numéro spécial de $L a$ Revue canadienne sur le vieillissement de mettre à jour leur travaux antérieurs. Bien que de nombreux auteurs étaient encore actifs dans le milieu universitaire, certains avaient pris leur retraite ou avaient changé d'occupations, et, malheureusement, les personnes suivantes sont décédées, et on les manque beaucoup : Dr William Forbes en 1999, Dr Ellen Gee en 2002 et Dr Kraus Arthur en 2009. Lorsque cela était possible, les articles dans ce numéro spécial de La Revue canadienne $d u$ vieillissement ont été écrites par les auteurs de la série originale de monographies et/ou par la génération des gérontologues sociaux actuelle et émérgeante au Canada. Des 15 monographies originales, 11 d'entre elles sont mises à jour. Les articles examinent les développements dans chacun des sous-domaines couverts par les monographies originales.

En outre, des articles ont été sollicités pour combler certaines lacunes non couvertes par la série originale 
de Butterworths. Quand la série de Butterworths a pris fin au début des années 1990, plusieurs monographies de plus aurait pu être écrites pour aborder des sujets qui étaient au centre de la gérontologie sociale, mais n'avaient pas encore été explorés. Par exemple, les grands absents étaient des monographies sur des constructions culturelles et les représentations du vieillissement, des activités de loisirs dans la vieillesse, l'état de santé et les transitions de santé à un âge plus avancé, et les questions à la fin de vie. En effet, il existe un besoin pour une série de monographies contemporaines et supplémentaires qui exploreraient des sujets négligés par la série originale et qui en outre traiteraient des domaines nouveaux et émergeants en gérontologie sociale.

Dans ce numéro spécial, 11 articles mettent à jour des monographies de la série originale et deux articles traitent des sujets nouvellement émergeants. Les thèmes relativement nouveaux comprennent un examen des travaux effectués sur les communautés amies des aînés - par Verena Menec, Robin Means, Norah Keating, Graham Parkhurst et Jacquie Eales - et un article sur le corps vieillissant par Laura Hurd Clarke et Alexandra Korotchenko. Ces domaines d'intérêt relativement nouveaux pour le monde académique sont ajoutés à des sujets abordés par la série originale et aident à compléter cette édition spéciale couvrante une diversité de sujets qui traitent de la gérontologie sociale contemporaine au Canada. Les experts dans chaque domaine specialisé ont examiné en détail chacun des articles acceptés pour ce numéro spécial.

Les monographies originales peuvent être regroupées en trois grandes catégories (voir la liste des références ci-dessous pour tous les auteurs et les titres originaux) :

1. La démographie, la géographie, et la diversité (le vieillissement dela population duCanada, la mobilitégéographique, le vieillissement au Canada rural, le vieillissement et l'ethnicité, les femmes et le veillissement, le veuvage);

2. La santé et soins de santé (l'institutionnalisation, les médicaments);

3. La securité et le bien-être (le soutien social, les liens familiaux, la securité économique, la retraite, la victimisation et la peur du crime, la violence envers les aînés, le bien-être psychologique) .

La série originale a exercé un impact considérable sur le développement de l'éducation gérontologique au Canada. Barry D. McPherson, rédacteur-en-chef général de la série de Butterworths, a écrit le premier manuel en gérontologie sociale au Canada. Son Aging as a Social Process a connu cinq éditions à ce jour $(1983,1990,1998$, 2004, 2008), et une sixième édition sera publiée en 2012. Pour la cinquième édition en 2008 et la prochaine edition sixième, Andrew Wister rejoint McPherson en tant que co-auteur. Un second manuel de gérontologie sociale intervient cinq ans apres le travail pionnier de McPherson. Aging and Society par Mark W. Novak a connu six éditions (1988, 1993, 2001, 2006 et 1010). Lori Campbell rejoint Novak en tant que co-auteur pour l'édition de 2001 et les suivantes.

Neena Chappell, Ellen Gee, Lynn McDonald, et Michael Stones, tous les auteurs de monographies dans la série originale de Butterworth, ont écrit le manuel de gérontologie sociale troisième pour le Canada (première édition en 2003 et deuxième édition en 2008). Malheureusement, Ellen Gee est décedée à la suite de la première édition.

En bref, la série de Butterworths a eu un impact considérable sur le développement de la gérontologie sociale et l'éducation en gérontologie au Canada.

Ce qui suit est une liste des monographies dans la série originale :

- Susan A. McDaniel. 1986. Canada's Aging Population.

- Leo Driedger and Neena Chappell, 1987. Aging and Ethnicity.

- William A. McKim and Brian L. Mishara, 1987. Drugs and Aging.

- Yves Brillon, 1987. Victimization and Fear of Crime among the Elderly.

- Ellen M. Gee and Meredith M. Kimball, 1987. Women and Aging.

- William F. Forbes, Jennifer A. Jackson, and Arthur S. Kraus, 1987. Institutionalization of the Elderly in Canada.

- Herbert C. Northcott, 1988. The Geographic Mobility of Elderly Canadians.

- Ingrid A. Connidis, 1989. Family Ties and Aging.

- P. Lynn McDonald and Richard A. Wanner, 1990. Retirement in Canada.

- Robert L. Brown, 1991. Economic Security in an Aging Population.

- P. Lynn McDonald, Joseph P. Hornick, Gerald B. Robertson, and Jean E. Wallace, 1991. Elder Abuse and Neglect in Canada.

- Anne Martin Matthews, 1991. Widowhood in Later Life.

- Albert Kozma, M.J. Stones, and J.K. McNeil, 1991. Psychological Well-Being in Later Life.

- Norah C. Keating, 1991. Aging in Rural Canada.

- Neena L. Chappell, 1992. Social Support and Aging.

Herbert C. Northcott et Mark W. Rosenberg

Rédacteurs invités 